\title{
Nonpharmacological interventions for the prevention of hypertension in low- and middle-income countries: a systematic review and meta-analysis
}

\author{
K. M. Saif-Ur-Rahman $\mathbb{1}^{1,2} \cdot$ Syed Shariful Islam ${ }^{2,3} \cdot$ Md Hasan $^{2,3} \cdot$ Shahed Hossain $^{1,4} \cdot$ Razib Mamun $^{1,2}$. \\ Sohana Shafique ${ }^{1} \cdot$ Al Mamun $^{1} \cdot$ Md. Khalequzzaman $^{2,3} \cdot$ Fariha Haseen $^{2,3} \cdot$ Aminur Rahman $^{1} \cdot$ Iqbal Anwar $^{1}$
}

Received: 22 January 2019 / Revised: 18 March 2019 / Accepted: 17 June 2019 / Published online: 20 August 2019

(c) The Author(s) 2019. This article is published with open access

\begin{abstract}
Hypertension is the single biggest cause of various cardiovascular complications and at the same time one of the most preventable phenomena. Low- and middle-income countries (LMICs) are facing increasing prevalence of hypertension which is imposing a huge burden on morbidity, premature mortality, and catastrophic health expenditure. This systematic review searched for the nonpharmacological interventions for prevention of hypertension among normotensive people in LMICs considering the period 1990-2016. This review has been conducted following standard methodology of Cochrane review involving two independent reviewers in screening, quality appraisal, and data extraction. Narrative synthesis of included articles was demonstrated using tables and meta-analysis was conducted to pool the estimates of studies which fulfilled the criteria. Total seven trials were included in the review with 6046 participants from eight LMICs. Two cluster randomized trials were pooled and there was a statistically significant effect (Systolic Blood Pressure: mean difference -2.35 [95\% CI: -4.31 to -0.38 ], Diastolic Blood Pressure: mean difference -2.11 [95\% CI: -3.20 to -1.02$]$ ) of home based health education in reducing blood pressure. Three individual studies reported reduction of blood pressure as a result of restricted dietary sodium intake. None of the studies was appraised as low risk of bias due to poor methodological quality. Non-pharmacological interventions can play important role in preventing the development of hypertension among normotensive people. Further trials with longer follow-up period and robust methods are recommended for getting stronger evidence on these interventions.
\end{abstract}

\section{Introduction}

Hypertension attributes to the $6 \%$ of global burden of diseases and is responsible for 7.7 million global premature deaths annually [1]. Hypertension and its complications alone causes $53 \%$ cardiovascular diseases related mortality

K. M. Saif-Ur-Rahman

su.rahman@icddrb.org

1 Health Systems and Population Studies Division, icddr,b, Dhaka, Bangladesh

2 Systematic Review Centre (SRC), Bangabandhu Sheikh Mujib Medical University (BSMMU), Dhaka, Bangladesh

3 Department of Public Health and Informatics, Bangabandhu Sheikh Mujib Medical University (BSMMU), Dhaka, Bangladesh

4 James P. Grant School of Public Health, BRAC University, Dhaka, Bangladesh which is in the long run responsible for almost one third of total annual deaths [2]. Due to the epidemiological transition from infectious diseases to noncommunicable diseases (NCDs) and unhealthy sedentary lifestyle, low- and middleincome countries (LMICs) are experiencing the increasing trend of hypertension prevalence [3]. A systematic review and meta-analysis estimated that the overall prevalence of hypertension in LMICs is $32.3 \%$ [4]. This phenomenon is contributing to the dual burden of both infectious diseases and NCDs in LMICs and at the same time imposing the catastrophic healthcare expenditure to the communities and the nations [5].

Despite highly prevalent, hypertension is one of the most preventable conditions [6, 7]. Various studies have demonstrated different lifestyle modification approaches to reduce or prevent hypertension. Recommended measures for preventing hypertension are reducing body weight in case of obesity, undertaking regular physical activity, reduced intake of salt or sodium, increasing potassium 
supplement, and avoiding harmful use of alcohol [8]. Dietary intervention such as dietary approaches to stop hypertension has also promisingly reduced blood pressure (BP) [9]. This specific dietary recommendation included more intake of vegetables and fruits, milk products with lower proportion of fat, reduction of cholesterol, and saturated fat in meals [9]. A systematic review synthesizing information from 16 intervention studies including $>3000$ participants reported the reduction of both systolic and diastolic BP with increased calcium intake. Reduction of BP was even higher with increasing dose of calcium. Effect of calcium in reducing BP was greater among the younger population [10]. Complementary and alternative medicine has been found useful sometimes in this aspect [11-13]. A systematic review incorporated six RCTs and explored the effectiveness of "Yoga" either alone or in combination with conventional therapies in lowering BP. Results from this review also positively associated in reduction of $\mathrm{BP}$ although the quality of the included trials was notified as low grade [11]. The mind body therapy which is a combination of physical exercise and meditation was examined by one review that included nine RCTs, 13 quasi experimental studies and 4 observational studies. Pooled estimation depicted the significant effect of "Tai Chi" - a martial art originating in ancient China, embracing the mind, body, and spirit—in reducing BP. Only few of the included RCTs were methodologically strong in this systematic review [12]. An overview of systematic review investigated the effect of transcendental meditation-a technique for reducing stress on BP. A total of eight systematic reviews including Cochrane reviews were included. Overall the assessment was fare in terms of the quality of the included reviews. Results from the overview also supported the role of meditation in lowering $\mathrm{BP}$ despite some conflicting results between included reviews [13]. Apart from dietary modifications, changing lifestyle, alternative medicine and meditation, therapeutic agent such as combination of Chlorthalidone and Amiloride have also been tested. This double blinded, placebo controlled randomized trial demonstrates the significance of the therapeutic agent in preventing hypertension [14]. Despite significant effect on prevention, there is risk of experiencing adverse effect among the participants. Taking regular medication for prevention may also raise question on compliance [15].

In addition, most of these interventions are based on high-income countries. LMICs are challenged with limited resources to provide useful programs for the early diagnosis, prevention, or control of this huge burden of disease $[2,7,16]$. Interventions for prevention of hypertension among persons with normal BP (Systolic BP (SBP) $120-139 \mathrm{mmHg}$ and diastolic BP (DBP) $80-89 \mathrm{mmHg}$ ) [17] or prehypertensive ( $\mathrm{SBP} \geq 120-139 \mathrm{mmHg}$ and/or DBP $\geq 80-89 \mathrm{mmHg}$ ) [18] can play an important role to tackle this progressively increasing disease and reduce complications and morbidity resulting from that. Our objective of the present systematic review is to explore all available interventions which are nonpharmacological in approach and to synthesize their effectiveness in prevention of hypertension in LMICs.

\section{Methods}

This systematic review has been carried out following the methodology of Cochrane systematic reviews [19] and addressed the requirements stated in preferred reporting items for systematic reviews and meta-analysis protocols guidelines [20, 21]. Details methodology including the development of search strategy, dual-screening process, dual-data extraction, dual appraisal of included articles for quality assessment, narrative synthesis, and meta-analysis has been described in the published protocol [22]. A comprehensive search strategy was developed using the key words such as Exercise, "Physical activity", "Weight loss", "Sodium restriction", "Dietary potassium", "Calcium supplementation", "Fish oil supplementation", Lifestyle, Hypertension, "Blood pressure" to search different electronic bibliographic database including Embase, MEDLINE through Pubmed, Web of Science, Clinical Trials. gov., the Cochrane Library (Cochrane Central Register of Controlled Trials (CENTRAL), Scopus etc. The search period covered from 1990 to 2016. Randomized control trials providing nonpharmacological intervention on normotensive adult population in LMICs were included. Both the screening of "title and abstract" and "full text" of the retrieved articles were conducted independently by two reviewers and any disagreement was resolved by a third reviewer. Reference management software was used to keep track of the screening process. Each of the studies was appraised critically for assessment of risk of bias (ROB). A narrative synthesis of the characteristics of study participants and types of intervention with specific outcome was demonstrated. Mean and standard deviation of both systolic and diastolic BP were recorded from baseline and endline information. For meta-analysis, a random effect model was chosen with 95\% confidence interval and both the chi squared and $\mathrm{I}^{2}$ statistic were measured. The systematic review is registered in International Prospective Register of Systematic Reviews. Registration number is CRD42017055423.

\section{Results}

A total of 5131 articles were retrieved after searching eight selected database using a comprehensive search strategy. 
After removing the duplicates, 4093 articles were compiled for title and abstract review. Applying inclusion and exclusion criteria, 19 articles were selected for full text review. We did not found the full text of four articles even after communicating with the corresponding author. The main causes of excluding these articles were irrelevance with the review objective. Out of these 15 articles, eight articles were excluded due to the following reasons: four articles were not focusing LMIC's; two articles did not follow RCT design; one article was a literature review and intervention was given on hypertensive population in one article. After screening, seven RCTs were included in the final analysis. The detailed description of the selection process of the included articles has been provided in Fig. 1 using PRISMA flow diagram.

\section{Quality assessment of the included studies}

We assessed the ROB of the included trials using the guideline of Cochrane review. Majority of the trials (five out of seven) performed the sequence generation randomly and reported accordingly. Only three articles described the process of allocation concealment. Thus, there was potential risk of selection bias in almost half of the trials. Only two studies maintained blinding at the level of participants and implementers. Another two trials mentioned about blinding at the level of outcome assessors. Overall, majority (five in each case) of the trials were unable to minimize the chance of performance bias and detection bias. Almost all the studies mentioned about attrition rate. Only one study did not describe regarding attrition and marked as unclear information. All the articles were with sufficient information regarding the primary outcome hence we recognized all of them as at low risk for selective reporting bias. Majority of the studies did not provide any information regarding

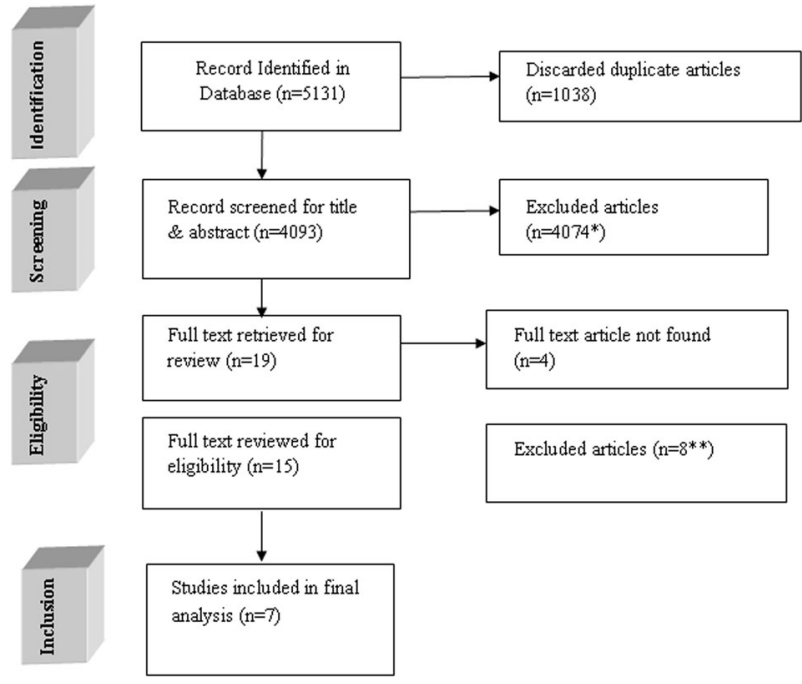

Fig. 1 Flow diagram of the inclusion process other potential biases. We did not get the description regarding contamination in cluster randomized trials and rationale behind the duration of washout period in the studies with crossover design. A graphical demonstration of assessment of ROB has been provided in Figs. 2, 3 .

A summary description of the basic characteristics of the included articles has been given in Table 1. Among the seven included RCTs, two studies used clustered randomized design [23, 24], two studies were randomized control trials $[25,26]$ and three studies were with crossover design [27-29]. Among the three crossover trials, two studies incorporated a washout period of 1 and 3 weeks, respectively $[28,29]$ before altering the treatment options. All the studies described the effectiveness of the intervention to prevent hypertension. The main outcome, BP, was measured manually with two exceptions which considered both manual and electronic measurements $[25,28]$. The interventions provided by different trials were health education program, soy drink and cow's milk; calcium tablets; and low- or high-salt intake. Among these studies only two met the criteria of meta-analysis and rest of the studies were

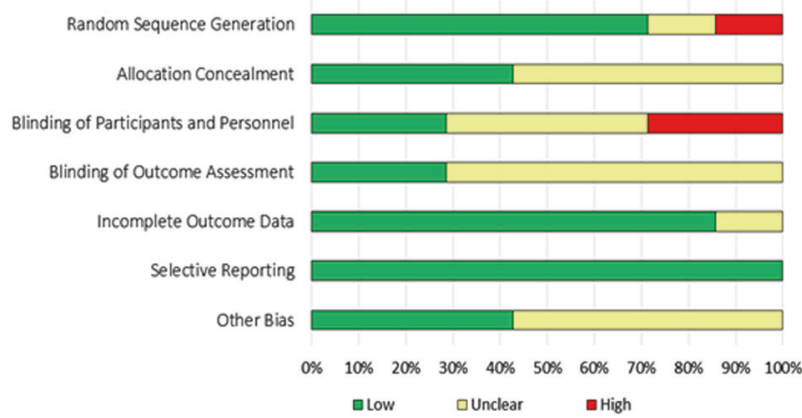

Fig. 2 Overall risk of bias among the included articles

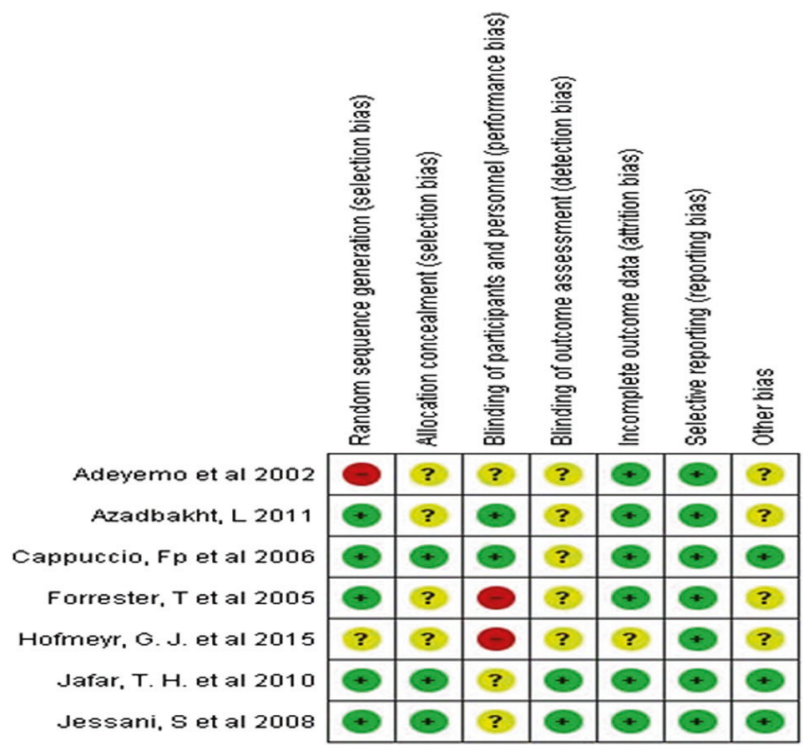

Fig. 3 Risk of bias assessment in individual studies 


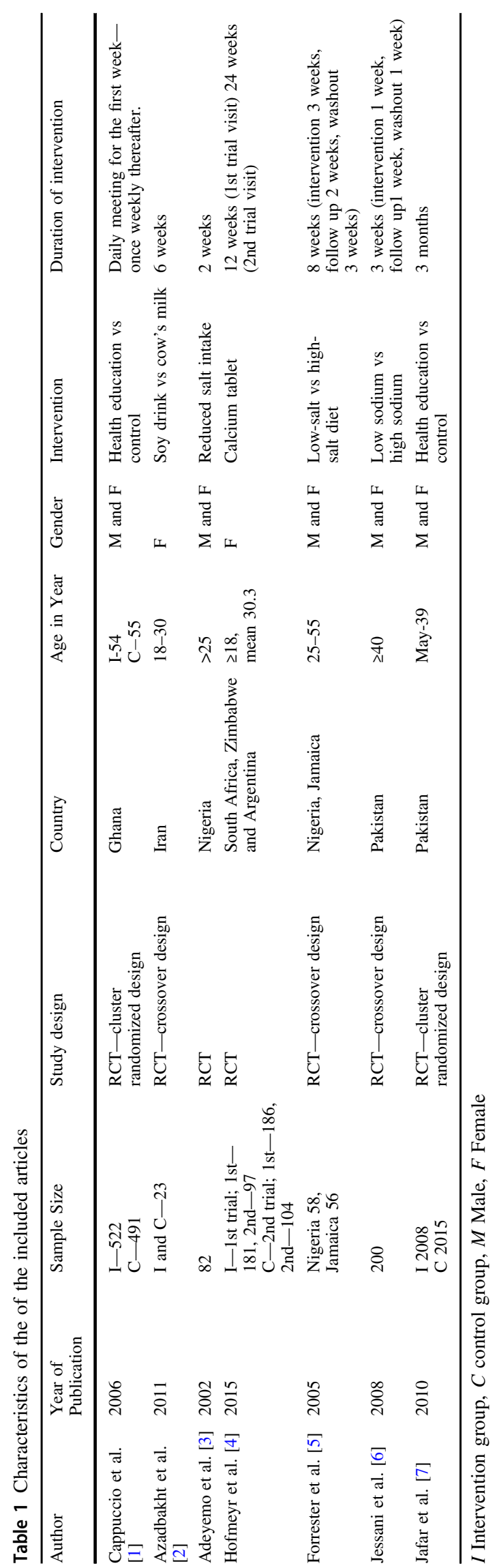

described using summary statistics. All the included studies considered both male and female except one [26] where intervention was provided among the nonpregnant women. Sample size of the studies varied based on the design adapted. Cluster randomized trials included large number of participants [23, 24] whereas some RCTs included $<100$ participants [25, 27]. Four studies were conducted in African region [23, 25, 26, 28]; one was in Middle East [27] and two studies were conducted in South Asia [24, 29]. All the studies included adult respondents and only one study included participants aged between 5 and 39 years [24].

Table 2 showed the results of studies that were not included in meta-analysis. These studies were heterogeneous enough for not including in the meta-analysis in terms of interventions, duration of the study, and study settings. Azadbakht et al. measured the beneficial effect of soy milk in comparison with cow's milk on anthropometric measurements and BP for overweight and obese female youths [27]. It showed that the SBP was reduced significantly following the soy drink intervention period than that of the cow's milk. Mean percent change during soy milk period was $-0.4 \pm 0.9$ and $-1.7 \pm 0.5$ during cow's milk period. Diastolic BP was also reduced in soy milk drink period $(-0.4 \pm 0.1$ vs $0.4 \pm 0.1)$. Adeyemo et al. provided dietary intervention among normotensive adults in South East Nigeria to determine the feasibility of reducing dietary sodium intake [25]. BP of the participants was measured using both manual procedure and electronic device. In manual measurement, among the participants on low sodium diet, SBP was reduced by $-4.7 \pm 2.8 \mathrm{mmHg}$ for men and by $-7 \pm 4.4 \mathrm{mmHg}$ for women. The diastolic BP was reduced by $-1.9 \pm 2.2 \mathrm{mmHg}$ among men and by $-1.6 \pm 3.4 \mathrm{mmHg}$ among women. Hofmeyr et al. explored the effect of calcium on the BP among nonpregnant women who experienced pre-eclampsia previously [26]. The follow-up was conducted in two phases at 12 weeks and 24 weeks, respectively. Participants were provided $500 \mathrm{mg}$ calcium tablet each day. Overall, the BP in calcium supplementation group was reduced but that was not statistically significant. Forrester et al. compared the effectiveness of high-salt diet (usual diet and additional $50 \mathrm{mEq}$ salt pills) and low-salt diet (usual diet and a reduction of 50 $\mathrm{mEq}$ salt) on BP [28]. This study was conducted in two regions of Nigeria and Jamaica and results were compared between these two zones as well. BP was measured using both electronic automated machine and manual procedure. The average result from the two procedures was used in the final analysis. The mean change in SBP between low and high sodium diet phase was $\sim 5 \mathrm{mmHg}$ in both groups. This study depicted that there is a significant efficacy of sodium reduction in lowering the BP. These results were consistent with the studies conducted among affluent population in high income countries. Jessani et al. also estimated the 
Table 2 Summary results of the studies not included in meta-analysis

\begin{tabular}{|c|c|c|c|c|c|c|c|c|c|c|c|c|c|}
\hline \multirow[t]{3}{*}{ Authors } & \multirow[t]{3}{*}{ Intervention } & \multicolumn{4}{|l|}{ SBP } & \multicolumn{4}{|l|}{ DBP } & \multicolumn{4}{|c|}{ Mean percent changes } \\
\hline & & \multicolumn{2}{|c|}{$\begin{array}{l}\text { Baseline/ } \\
\text { placebo }\end{array}$} & \multicolumn{2}{|c|}{$\begin{array}{l}\text { Endline/ } \\
\text { intervention }\end{array}$} & \multicolumn{2}{|c|}{$\begin{array}{l}\text { Baseline/ } \\
\text { placebo }\end{array}$} & \multicolumn{2}{|c|}{$\begin{array}{l}\text { Endline/ } \\
\text { intervention }\end{array}$} & \multicolumn{2}{|l|}{ SBP } & \multicolumn{2}{|l|}{ DBP } \\
\hline & & Mean & SD & Mean & SD & Mean & SD & Mean & SD & Mean & SE & Mean & SE \\
\hline \multicolumn{14}{|l|}{ Azadbakht et al. [2] } \\
\hline & Cow's milk & 102.1 & 2.2 & 100 & 2.1 & 65.6 & 2.2 & 65.8 & 2 & -1.7 & 0.5 & 0.4 & 0.1 \\
\hline & Soy milk & 100.8 & 2.1 & 96 & 2.2 & 66.3 & 2 & 66 & 2.2 & -4 & 0.9 & -0.4 & 0.1 \\
\hline Adeyemo et al. [3] & $\begin{array}{l}\text { Manual Low vs high sodium } \\
\text { diet-women }\end{array}$ & 110.1 & 14.6 & 103.1 & 9.8 & 69.1 & 11.5 & 67.5 & 7.5 & -7 & 4.4 & -1.6 & 3.4 \\
\hline Adeyemo et al. [3] & Low vs high sodium diet-men & 116.8 & 15.3 & 112.1 & 12.5 & 74 & 9.5 & 72.1 & 9.5 & -4.7 & 2.8 & -1.9 & 2.2 \\
\hline \multirow[t]{2}{*}{ Hofmeyr et al. [4] } & $\begin{array}{l}\text { 1st trial—Placebo vs Calcium } \\
\text { supplementation }\end{array}$ & 126.1 & 16.3 & 127.4 & 17.2 & 81.6 & 11.5 & 81.5 & 13 & 1.4 & 3 & 1 & 2.4 \\
\hline & $\begin{array}{l}\text { 2nd trial-Placebo vs Calcium } \\
\text { supplementation }\end{array}$ & 126.8 & 16 & 131 & 19.6 & 81.9 & 11.4 & 83.6 & 14.5 & 2.5 & 4.4 & 1.4 & 3.5 \\
\hline \multirow[t]{2}{*}{ Forrester et al. [5] } & $\begin{array}{l}\text { Nigeria-high vs low } \\
\text { sodium phase }\end{array}$ & 114.8 & 11.4 & & & 73.3 & 9.1 & & & 4.5 & 2.9 & 2.7 & 2 \\
\hline & $\begin{array}{l}\text { Jamaica-high vs low } \\
\text { sodium phase }\end{array}$ & 122.3 & 10.2 & & & 75.9 & 7.3 & & & 5.5 & 1.5 & 2.8 & 2.3 \\
\hline Jessani et al. [6] & High sodium phase & 122 & 11 & 134 & 40 & 79 & 6 & 82 & 13.5 & 6 & 3 & 0 & 1 \\
\hline High normal SBP & Low sodium phase & 122 & 11 & 128 & 20 & 79 & 6 & 81 & 13.5 & & & & \\
\hline Jessani et al. [6] & High sodium phase & 122 & 11 & 118 & 27 & 79 & 6 & 82 & 13.5 & -1 & 1 & 0 & 1 \\
\hline Normal SBP & Low sodium phase & 122 & 11 & 119 & 27 & 79 & 6 & 81 & 13.5 & & & & \\
\hline
\end{tabular}

effect of high and low sodium diet among the Pakistani population [29]. Participants were randomly allocated either in a low sodium diet group $(20 \mathrm{mEq} /$ day $)$ or a high sodium diet group (220 mEq/day) for 1 week. The washout period for both the groups was 1 week before the crossover phase. The crossover period with the altered diet was for another 1 week. Researchers measured the difference in SBP and diastolic BP in each phase as primary outcome. SBP was classified as high normal SBP and normal SBP. The result showed that adjusted mean of SBP reduced significantly among the participant with high normal SBP (130-139 $\mathrm{mmHg}$ ) during low sodium diet in comparison with baseline. There were no significant changes among the participants with normal SBP $(<130 \mathrm{mmHg})$ at the baseline.

\section{Meta-analysis}

In this analysis, only two articles fulfilled the criteria of meta-analysis. Pooled estimate revealed a significant reduction in $\mathrm{BP}$ of home based health education with control (pooled mean difference SBP: -2.35 [95\% CI -4.31 , $-0.38] \mathrm{mmHg}, p=0.02$; pooled mean difference DBP: -2.11 [95\% CI -3.20, - 1.02] mmHg, $p=0.0001$ ) (Fig. 4). Heterogeneity between these cluster randomized trials was very low (SBP: $I^{2}=18 \%$; DBP: $I^{2}=0 \%$ ) which implies that there is necessity to conduct meta-analysis without subgroup analysis. The forest plot is demonstrated in Fig. 4.

\section{Publication bias}

In our review, it was not possible to observe the publication bias because of very few numbers of included studies for meta-analysis. Funnel plot generally used to estimate the risk of publication bias. For only two studies, result of this graph is unpredictable. It is also recommended that test of funnel plot asymmetry or existence of publication bias is not possible if the selected study is $<10$ in meta-analysis [30].

\section{Discussion}

With an aim to examine the effectiveness of nonpharmacological interventions for prevention of hypertension in LMICs, this systematic review included seven trials incorporating 6046 patients from eight countries over the last 16 years. Although a comprehensive search was undertaken, just seven studies met the inclusion criteria. The studies investigated a range of interventions on normotensive population including health education, soy drink, calcium supplementation, and low sodium diet. Only of two studies were eligible to [23, 24] combine the 
Fig. 4 Comparison between home health education (HHE) mean difference of systolic blood pressure and diastolic blood pressure and no HHE, outcome effect
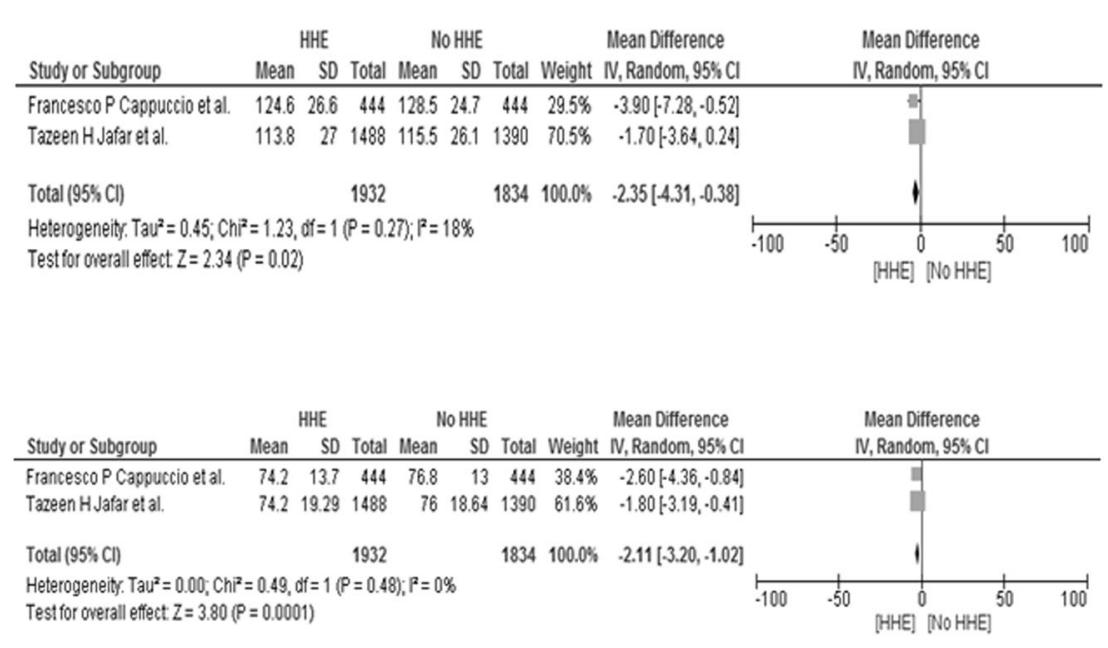

outcomes through meta-analysis which showed the effects of health education on reducing BP in normotensive participants. The significant change in BP indicates that positive effect of health education among normotensive individuals in reducing their BP. However, there were dissimilarities in number and age group among two study participants. Health education for the prevention of hypertension is widely used in the interventions which covered prehypertension individuals. Moreover, this approach is also used for normotensive individuals to make them aware. Study showed that community-based health education program on hypertension and cardiovascular risk factors resulted into declining BP of the participants in the intervention group [31]. The behavior change communication messages mentioned in the included articles in this review focused on knowledge of lifestyle modification such as lowering salt intake in food [23], increased physical activity, cessation of smoking, consumption of low fat diet including dairy products, and increased intake of fruits and vegetables [24]. Another systematic review demonstrated the effectiveness of lifestyle modification on metabolic syndrome where information from eight trials were pooled together [32].

In this review, three studies reported effect on reducing BP through reduction in dietary sodium intake [25, 28, 29]. Intervention period in these studies were short, ranging from 2 to 8 weeks. The result showed significant effect of sodium intake modification, it is worth mentioning that the three included studies varied in their findings, reporting different range of changes in SBP and DBP. A Cochrane review also demonstrated the significant change in $\mathrm{BP}$ among White, Black, and Asian people with normal BP where there was a greater reduction rate among Black and Asian people [33].

One RCT (crossover design) focused on the effect of soy drinks and cow milk among the females [27]. SBP reduced significantly but no significant changes in DBP due to the intervention of soy drink. However, included participants were obese and on a weight reducing diet. Calcium tablet was given to intervention group in one study [26] where no significant change in reducing BP was observed. Similar results has been demonstrated in a systematic review incorporating sixteen trials [10] where slight reduction of BP was observed due to increase in dietary calcium intake. However, these trials could not conclude with strong recommendations for this specific intervention.

As per world health organization, sodium reduction in food intake can reduce BP in normotensive individuals [2]. In this systematic review, a trend toward reduction in BP was observed by dietary sodium reduction. Studies conducted by Law et al. and Miller et al. [34, 35] reveled that participants having higher $\mathrm{BP}$ at baseline resulted in greater reductions in BP due to the effect of sodium reduction. Many studies demonstrated that decreasing sodium in diets have benefits in lowering BP among prehypertensive participants [36-38]. However, one study showed that the reduction of dietary sodium has no significant effect in normotensive individuals with $\mathrm{SBP}<130 \mathrm{mmHg}$ and with normal kidney function [39]. Another crossover study reported no significant changes in BP due to the reduction of salt intake [40].

No studies on physical activities related intervention to reduce hypertension was found in LMICs. However, metaanalysis of seven studies conducted among Brazilian population also demonstrated the significant impact of resistance exercise and aerobics in lowering both SBP and DBP [41]. Pooled estimate showed reduction in both systolic and diastolic pressures which was statistically significant. But the sample size of included studies were very small with short intervention period and both normotensive and hypertensive patients were included. Another systematic review and meta-analysis conducted demonstrated 
that isometric handgrip exercise is efficacious for reducing SBP and DBP in adult participants [42].

We conducted a broad search of several databases but placed restrictions on the language of the study when searching the electronic databases. Studies published in english language were only considered in this review which is one of the main limitations. It is likely that there are other studies published in other languages which we have missed in this review. Strengths of this systematic review are the inclusion of RCTs only and following Cochrane guideline strictly.

None of the included studies was found as low ROB and also methodologically none of the studies was of high quality. In some studies, short duration of intervention period with small sample demonstrated quick significant results but their sustainability remained questionable. Follow-up after a longer duration could have observed for the sustainability of the impact of interventions. Studies did not mention about potential confounders and contamination in case of cluster randomized trials.

\section{Conclusion}

This review demonstrated the effectiveness of nonpharmacological interventions for prevention of hypertension of the included trials. Given the limited scientific evidence in LMICs and quality of the evidences, no strong conclusion about effectiveness of nonpharmacological approaches could be drawn. This systematic review highlights the need of future research opportunities and a necessity for more scientific studies with larger numbers of participants and longer intervention period using robust study design.

\section{Summary}

\section{What is known about topic}

- Hypertension attributes to the cardiovascular disease burden and responsible for premature death.

- Different non pharmacological approaches have been implemented for prevention of hypertension in high income countries

\section{What this study adds}

- Explored all available non pharmacological intervention trials in LMICs

- Home based health education has got significant effect in preventing hypertension among people with normal blood pressure in LMICs
- More researches with robust methodology and longer follow up are required for recommending other non pharmacological approaches

\section{Data availability}

The datasets generated and/or analyzed during in this review shall be available from the corresponding author on reasonable request.

Funding There is no external funding for this systematic review. This review has been conducted by the Systematic Review Centre (SRC) of Department of Public Health and Informatics at Bangabandhu Sheikh Mujib Medical University (BSMMU), Bangladesh which has been established with the support of SHARE (Strengthening Health Applying Research Evidence) project of icddr,b funded by the European Union (Grant Contract No DCI-SANTE/2014/342-479). icddr,b acknowledges with gratitude the commitment of European Union (EU) to its research efforts. icddr,b is also grateful to the Governments of Bangladesh, Canada, Sweden and the UK for providing core/unrestricted support.

Author contributions KMSUR, IA, SH, MH, and SI conceptualized the review in consultation with the co-reviewers. KMSUR wrote the first draft of this manuscript with substantial inputs from MH and RM. KMSUR, MH, and AM performed to the literature search. Screening, collection, and analysis of data for all the included interventions were conducted by KMSUR and MH with close consultation from SH, RM, SS, SI, AR, MK, FH, and IA. All authors provided input, reviewed and finalized the paper before dissemination. The corresponding author is the guarantor of this review. All authors read and approved the final manuscript.

\section{Compliance with ethical standards}

Conflict of interest The authors declare that they have no conflict of interest.

Ethics and dissemination Approval for conducting this systematic review has been taken from the Institutional Review Board (IRB) of Bangabandhu Sheikh Mujib Medical University (BSMMU). No additional formal ethical assessment and no informed consent are required. Findings of the systematic review will be published in international peer reviewed journal for dissemination.

Publisher's note: Springer Nature remains neutral with regard to jurisdictional claims in published maps and institutional affiliations.

Open Access This article is licensed under a Creative Commons Attribution 4.0 International License, which permits use, sharing, adaptation, distribution and reproduction in any medium or format, as long as you give appropriate credit to the original author(s) and the source, provide a link to the Creative Commons license, and indicate if changes were made. The images or other third party material in this article are included in the article's Creative Commons license, unless indicated otherwise in a credit line to the material. If material is not included in the article's Creative Commons license and your intended use is not permitted by statutory regulation or exceeds the permitted use, you will need to obtain permission directly from the copyright holder. To view a copy of this license, visit http://creativecommons. org/licenses/by/4.0/. 


\section{References}

1. Forouzanfar MH, Liu P, Roth GA, Ng M, Biryukov S, Marczak L, et al. Global burden of hypertension and systolic blood pressure of at least 110-115 mmhg, 1990-2015. JAMA. 2017;317:165-82.

2. WHO. A global brief on hypertension: silent killer, global public health crisis: World Health Day 2013. World Health Organization. 2013.

3. Mackay J, Mensah G, Mendis S, Greenlund K. The atlas of heart disease and stroke. Geneva, Switzerland: World Health Organization; 2004.

4. Sarki AM, Nduka CU, Stranges S, Kandala N-B, Uthman OA. Prevalence of hypertension in low-and middle-income countries: a systematic review and meta-analysis. Medicine. 2015;94:e1959.

5. Si Y, Zhou Z, Su M, Ma M, Xu Y, Heitner J. Catastrophic healthcare expenditure and its inequality for households with hypertension: evidence from the rural areas of Shaanxi Province in China. Int J Equity Health. 2017;16:27.

6. Kearney PM, Whelton M, Reynolds K, Muntner P, Whelton PK, He J. Global burden of hypertension: analysis of worldwide data. Lancet. 2005;365:217-23.

7. Lim SS, Vos T, Flaxman AD, Danaei G, Shibuya K, AdairRohani $\mathrm{H}$, et al. A comparative risk assessment of burden of disease and injury attributable to 67 risk factors and risk factor clusters in 21 regions, 1990-2010: a systematic analysis for the Global Burden of Disease Study 2010. Lancet. 2012;380: 2224-60.

8. Slama M, Susic D, Frohlich ED. Prevention of hypertension. Curr Opin Cardiol. 2002;17:531-6.

9. Appel LJ, Brands MW, Daniels SR, Karanja N, Elmer PJ, Sacks FM. Dietary approaches to prevent and treat hypertension: a scientific statement from the American Heart Association. Hypertens. 2006;47:296-308.

10. Cormick G, Ciapponi A, Cafferata ML, Belizan JM. Calcium supplementation for prevention of primary hypertension. Cochrane Database Syst Rev. 2015;30:Cd010037.

11. Wang J, Xiong X, Liu W. Yoga for essential hypertension: a systematic review. PLoS ONE. 2013;8:e76357.

12. Yeh GY, Wang C, Wayne PM, Phillips RS. The effect of tai chi exercise on blood pressure: a systematic review. Prev Cardiol. 2008;11:82-9.

13. Ooi SL, Giovino M, Pak SC. Transcendental meditation for lowering blood pressure: an overview of systematic reviews and meta-analyses. Complement Ther Med. 2017;34:26-34.

14. Fuchs SC, Poli-de-Figueiredo CE, Figueiredo Neto JA, Scala LCN, Whelton PK, Mosele F, et al. Effectiveness of chlorthalidone plus amiloride for the prevention of hypertension: the PREVER-prevention randomized clinical trial. J Am Heart Assoc. 2016;5:e004248.

15. Sabaté E. Adherence to long-term therapies: evidence for action. Switzerland: World Health Organization; 2003.

16. Ibrahim MM, Damasceno A. Hypertension in developing countries. Lancet. 2012;380:611-9.

17. Gabb GM, Mangoni A, Anderson CS, Cowley D, Dowden JS, Golledge J, et al. Guideline for the diagnosis and management of hypertension in adults-2016. Med J Aust. 2016;205:85-9.

18. Chia Y. Prehypertension: what Is the current status? Malays Fam Physician. 2008;3:72.

19. Higgins J, Green S. Cochrane handbook for systematic reviews of interventions version 5.1.0. England: The Cochrane Collaboration; 2011.

20. Moher D, Shamseer L, Clarke M, Ghersi D, Liberati A, Petticrew $\mathrm{M}$, et al. Preferred reporting items for systematic review and metaanalysis protocols (PRISMA-P) 2015 statement. Syst Rev. 2015;4:1.
21. Shamseer L, Moher D, Clarke M, Ghersi D, Liberati A, Petticrew $\mathrm{M}$, et al. Preferred reporting items for systematic review and metaanalysis protocols (PRISMA-P) 2015: elaboration and explanation. BMJ. 2015;349:g7647.

22. Saif-Ur-Rahman KM, Hasan M, Hossain S, Shafique S, Khalequzzaman $\mathrm{M}$, Haseen $\mathrm{F}$, et al. Non-pharmacological interventions for the prevention of hypertension in low-income and middleincome countries: protocol for a systematic review and metaanalysis. BMJ Open. 2018;8:e020724.

23. Cappuccio FP, Kerry SM, Micah FB, Plange-Rhule J, Eastwood JB. A community programme to reduce salt intake and blood pressure in Ghana [ISRCTN88789643]. BMC Public Health. 2006;6:13.

24. Jafar TH, Islam M, Hatcher J, Hashmi S, Bux R, Khan A, et al. Community based lifestyle intervention for blood pressure reduction in children and young adults in developing country: cluster randomised controlled trial. BMJ. 2010;340:c2641.

25. Adeyemo AA, Prewitt TE, Luke A, Omotade O, Rotimi CN, Brieger $\mathrm{W}$, et al. The feasibility of implementing a dietary sodium reduction intervention among free-living normotensive individuals in south west Nigeria. Ethn Dis. 2002;12:207-12.

26. Hofmeyr G, Seuc A, Betrán A, Purnat T, Ciganda A, Munjanja S, et al. The effect of calcium supplementation on blood pressure in non-pregnant women with previous pre-eclampsia: an exploratory, randomized placebo controlled study. Pregnancy Hypertens. 2015;5:273-9.

27. Azadbakht L, Nurbakhsh S. Effect of soy drink replacement in a weight reducing diet on anthropometric values and blood pressure among overweight and obese female youths. Asia Pac J Clin Nutr. 2011;20:383-9.

28. Forrester T, Adeyemo A, Soarres-Wynter S, Sargent L, Bennett F, Wilks $\mathrm{R}$, et al. A randomized trial on sodium reduction in two developing countries. J Hum Hypertens. 2005;19:55-60.

29. Jessani S, Hatcher J, Chaturvedi N, Jafar TH. Effect of low vs. high dietary sodium on blood pressure levels in a normotensive Indo-Asian population. Am J Hypertens. 2008;21:1238-44.

30. Ioannidis JP, Trikalinos TA. The appropriateness of asymmetry tests for publication bias in meta-analyses: a large survey. CMAJ. 2007;176:1091-6.

31. Fortmann SP, Winkleby MA, Flora JA, Haskell WL, Taylor CB. Effect of long-term community health education on blood pressure and hypertension control: the stanford five-city project. Am J Epidemiol. 1990;132:629-46.

32. Yamaoka K, Tango T. Effects of lifestyle modification on metabolic syndrome: a systematic review and meta-analysis. BMC Med. 2012;10:138.

33. Graudal NA, Hubeck-Graudal T, Jurgens G. Effects of low sodium diet versus high sodium diet on blood pressure, renin, aldosterone, catecholamines, cholesterol, and triglyceride. Cochrane database Syst Rev. 2017;4:Cd004022.

34. Law M, Frost C, Wald N. By how much does dietary salt reduction lower blood pressure? III-analysis of data from trials of salt reduction. BMJ. 1991;302:819-24.

35. Miller JZ, Daugherty SA, Weinberger MH, Grim CE, Christian JC, Lang CL. Blood pressure response to dietary sodium restriction in normotensive adults. Hypertens. 1983;5:790-5.

36. Cook NR, Cutler JA, Obarzanek E, Buring JE, Rexrode KM, Kumanyika SK, et al. Long term effects of dietary sodium reduction on cardiovascular disease outcomes: observational follow-up of the trials of hypertension prevention (TOHP). BMJ. 2007;334:885.

37. Vasan RS, Sullivan LM, Wilson PW, Sempos CT, Sundström J, Kannel WB, et al. Relative importance of borderline and elevated levels of coronary heart disease risk factors. Ann Intern Med. 2005;142:393-402.

38. Whelton PK, Appel L, Charleston J, Dalcin AT, Ewart C, Fried L, et al. The effects of nonpharmacologic interventions on blood 
pressure of persons with high normal levels: results of the Trials of Hypertension Prevention, phase I. JAMA. 1992;267:1213-20.

39. Todd AS, Macginley RJ, Schollum JB, Williams SM, Sutherland WH, Mann JI, et al. Dietary sodium loading in normotensive healthy volunteers does not increase arterial vascular reactivity or blood pressure. Nephrology. 2012;17:249-56.

40. Dickinson KM, Clifton PM, Keogh JB. A reduction of $3 \mathrm{~g} /$ day from a usual $9 \mathrm{~g}$ /day salt diet improves endothelial function and decreases endothelin-1 in a randomised cross_over study in normotensive overweight and obese subjects. Atherosclerosis. 2014;233:32-8.

41. Bento VFR, Albino FB, Moura KFd, Maftum GJ, Santos MdCd, Guarita-Souza LC, et al. Impact of physical activity interventions on blood pressure in Brazilian populations. Arq Bras de Cardiol. 2015;105:301-8.

42. Kelley GA, Kelley KS. Isometric handgrip exercise and resting blood pressure: a meta-analysis of randomized controlled trials. J Hypertens. 2010;28:411-8. 